\section{ORIGINAL RESEARCH}

M. Ishiyama

S. Fuwa

Y. Numaguchi

N. Kobayashi

Y. Saida

\title{
Pedicle Involvement on MR Imaging Is Common in Osteoporotic Compression Fractures
}

BACKGROUND AND PURPOSE: Pedicle involvement on MR imaging has been considered specific for malignancy. However, we also noted the findings in many patients with osteoporosis and hypothesized that it is not specific for malignant lesions. The aim of this study was to evaluate the prevalence of pedicle involvement in painful osteoporotic compression fractures and to determine whether the sign is specific for malignancy.

MATERIALS AND METHODS: We retrospectively reviewed MR images and CT scans of 152 patients who underwent PV for painful compression fractures. There were 140 patients ( 225 vertebrae) with osteoporotic fractures and 12 patients (19 vertebrae) with malignant fractures. Three radiologists evaluated the degree and extent of signal-intensity changes of the pedicle on MR imaging by consensus. The CT findings were also evaluated. The $\chi^{2}$ test was used for statistical analyses.

RESULTS: Of the 225 vertebrae of osteoporotic fractures and 19 vertebrae of malignant fractures, pedicle involvement on MR imaging was seen in 144 (64\%) and 16 (84.2\%) vertebrae, respectively, and there was no statistically significant difference $(P=.065)$. Positive pedicle involvement in osteoporotic fractures was seen in $84(77 \%)$ of 109 vertebrae with early-phase fractures ( $\leq 3$ months) and $60(51.7 \%)$ of 116 vertebrae with chronic-phase fractures ( $>3$ months), and this was statistically significant $(P<.001)$. Among 144 osteoporotic vertebrae that showed positive pedicle involvement on MR imaging, 45 (31\%) showed pedicle fractures and 55 (38.2\%) showed sclerotic change on CT.

CONCLUSIONS: Pedicle involvement was seen frequently in patients with osteoporotic compression fractures and was not specific for malignancy in our study group.

ABBREVIATIONS: Gd-T1WI = gadolinium-enhanced T1WI; PV = percutaneous vertebroplasty; STIR = short-tau inversion recovery; T1WI = T1-weighted imaging; T2WI = T2-weighted imaging

D ifferentiation of benign osteoporotic compression fractures and malignant pathologic fractures is clinically important, particularly in the elderly, but is sometimes difficult. MR imaging and CT have been shown to be helpful in differentiating both conditions. Compression fractures due to malignant tumors have a convex posterior cortex of the vertebral bodies, epidural or paravertebral masses, or infiltration of the posterior elements. ${ }^{1-5}$ Of these, pedicle involvement has been described as specific for malignant lesions. ${ }^{3}$ Osteoporotic compression fractures usually show retropulsion of a posterior bone fragment or intravertebral fluid. ${ }^{2,3,5,6}$

In our experience of $\mathrm{PV}$ for painful compression fractures, abnormal signal-intensity change in the pedicle on MR imaging, which will be defined as "pedicle involvement," has commonly been noted not only in patients with malignant compression fractures but also in those with benign osteoporotic compression fractures, and differentiation by using only this sign is sometimes difficult.

The aim of this study was to evaluate the prevalence and features of pedicle-involvement change on MR imaging in painful osteoporotic compression fractures and to determine whether these findings are truly specific for malignancy.

Received August 4, 2009; accepted August 24.

From the Department of Radiology, St. Luke's International Hospital, Tokyo, Japan.

Please address correspondence to Mitsutomi Ishiyama, MD, Department of Radiology, St. Luke's International Hospital, 9-1 Akashi-Cho, Chuo-Ku, Tokyo, Japan; e-mail: mitishi@ luke.or.jp

DOI 10.3174/ajnr.A1905

\section{Materials and Methods}

All protocols for this retrospective Health Insurance Portability and Accountability Act-compliant study were approved by the institutional review board. The need for informed consent was waived.

\section{Subjects}

We retrospectively reviewed medical records and MR imaging/CT data from 200 consecutive patients who had undergone PV for painful osteoporotic or malignant vertebral fractures. Patients who underwent full imaging studies (plain CT, T1WI, STIR imaging, and GdT1WI) were included. Exclusion criteria were the following: an insufficient imaging study, traumatic compression fracture without osteoporosis, PV for sacral fractures or Schmorl nodes, and repeat PV for vertebrae that had already undergone PV. The final study group comprised 140 patients with osteoporotic compression fractures ( 225 vertebrae; 35 women, 105 men; mean age, 77.8 years; range, 55-96 years) and 12 patients with malignant fractures (19 vertebrae; 7 women, 5 men; mean age, 61 years; range, $46-71$ years). Diagnosis was primarily made on the basis of medical history, thorough physical examination, laboratory findings, and imaging studies, including plain radiography, $\mathrm{CT}$, and MR imaging. When there were the characteristic findings indicating a benign process such as retropulsion of the posterior bone fragment or intravertebral cleft without bone destruction, the diagnosis was a benign compression fracture.

The final diagnosis of benign compression fracture was made by means of follow-up plain radiography and clinical history at 3 months and 1 year after PV. When the appearance of a follow-up radiographic study did not change significantly and no new malignancy was found, the fracture was considered to be caused by a benign process. Biopsy was performed to rule out malignant lesions for 3 patients with osteoporosis with a history of malignancy. When there was an intra- or 

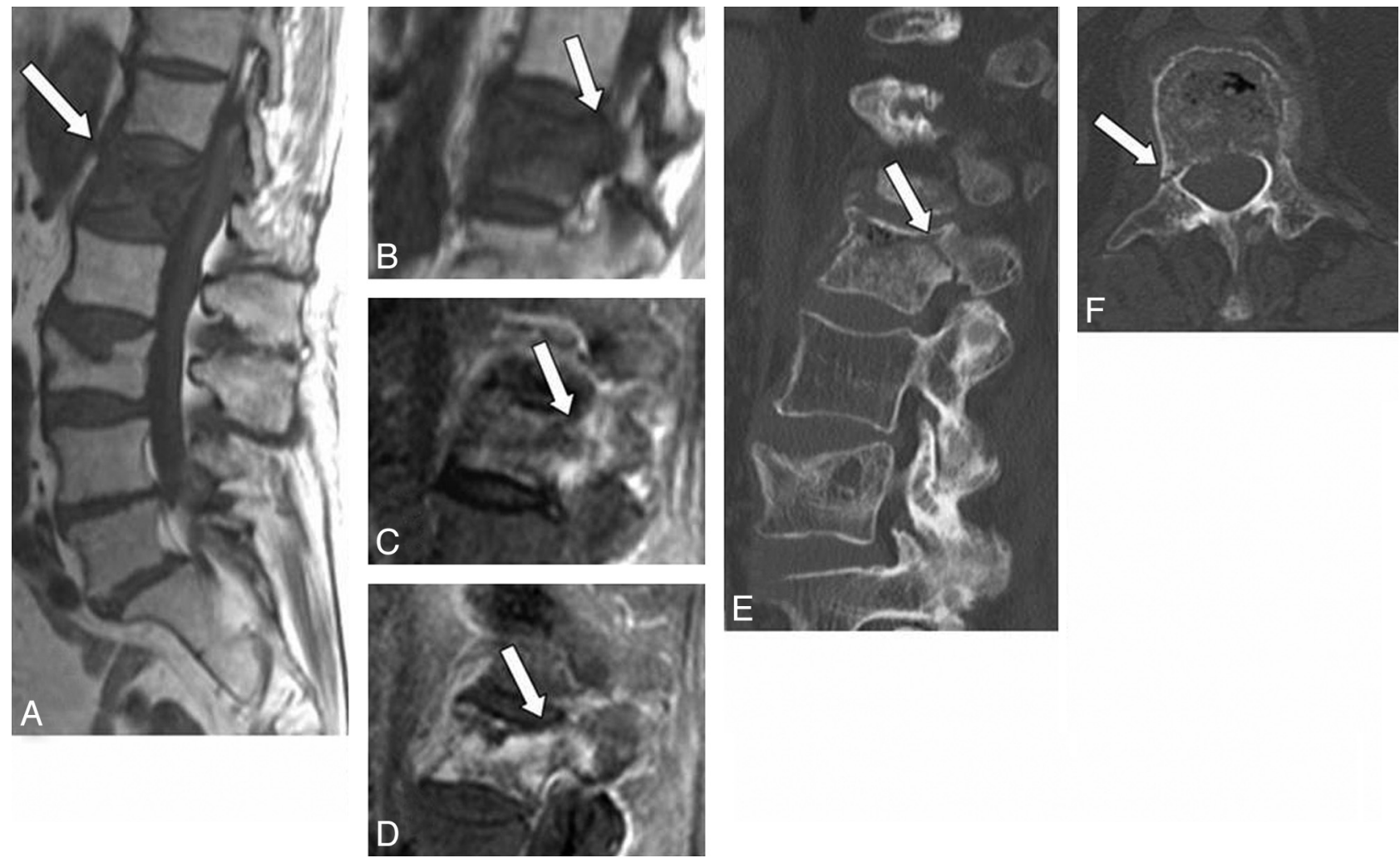

Fig 1. A 79-year-old woman with benign compression fracture which occurred one-and-a-half months earlier. $A$ and $B$, T1WI shows diffuse hypointensity in the vertebral body and the right pedicle of $\mathrm{L} 1$ (arrow). C, STIR image shows heterogeneous hyperintensity in these areas (arrow). D, Contrast-enhanced T1WI shows marked enhancement (arrow). E and $F$, On sagittal $(E)$ and axial ( $P)$ CT scans, fracture is seen in the right pedicle (arrow), which is a presumable reason for the abnormal signal intensity on MR imaging.

paravertebral mass or cortical bone destruction in the fractured vertebra, it was diagnosed as a malignant pathologic fracture. Among the 12 patients with malignancy, 11 patients had metastatic carcinoma (bronchogenic carcinoma, $n=5$; prostate carcinoma, $n=2$; breast carcinoma, $n=1$; vaginal carcinoma, $n=1$; pancreatic carcinoma, $n=1$; and cervical carcinoma, $n=1$ ), and 1 patient had multiple myeloma.

\section{Imaging}

MR imaging of the thoracolumbar spine was performed with 1 of two 1.5T scanners (Signa Excite, GE Healthcare, Milwaukee, Wisconsin; or Intera Achieva, Philips Medical Systems, Best, the Netherlands) by using a spine-array surface coil. The imaging protocol included sagittal spin-echo T1WI (TR/TE $=474-550 / 10.5-13 \mathrm{~ms})$, STIR images $(\mathrm{TR} / \mathrm{TE} / \mathrm{TI}=2500-6000 / 70-93 / 150 \mathrm{~ms})$, and fat-saturated spinecho Gd-T1WI $\left(\mathrm{TR} / \mathrm{TE}=460-600 / 6.2-12\right.$; flip angle $=80^{\circ}-90^{\circ} ; 0.2$ $\mathrm{mmol} / \mathrm{kg}$ ) with a $4-\mathrm{mm}$ section thickness and $0.5-\mathrm{mm}$ section spacing. The matrices were $512 \times 306$ and $512 \times 384$ with an FOV of 480 $\mathrm{mm}$ or $320 \times 224$ with an FOV of $280 \mathrm{~mm}$ (divided into 2 series). In some cases, axial images were obtained on Gd-T1WI, which were not evaluated in this study.

CT was also performed by using a 64 -section scanner or a 16 section scanner. Reconstructed axial, transverse, and sagittal images were obtained with a $3-\mathrm{mm}$ section thickness. The matrix was $512 \times$ 512 .

\section{Image Analysis}

Three radiologists evaluated the degree and extent of signal-intensity changes of the pedicle on MR imaging by consensus. Signal-intensity changes were classified as iso-, hyper-, or hypointense to the marrow of normal unaffected pedicles in the same patient on T1WI, iso- or hyperintense on STIR images and as showing mild or marked enhancement on Gd-T1WI. Pedicle involvement was defined as positive when at least 2 of 3 sequences showed abnormal signal-intensity change in the pedicle. The extent of the pedicle involvement was classified as partial or diffuse. CT findings of the pedicle were categorized into 3 groups: fracture (Fig 1), sclerosis (Fig 2), or no apparent abnormality (Fig 3)

\section{Statistical Analysis}

The $\chi^{2}$ test was used for statistical analyses, with values of $P<.05$ considered significant.

\section{Results}

Pedicle involvement on MR imaging in benign osteoporotic compression fractures and malignant pathologic fractures is shown in Table 1 . Of the 225 vertebrae of osteoporotic fractures and 19 vertebrae of malignant pathologic fractures, pedicle involvement was seen in 144 (64\%) and $16(84.2 \%)$ vertebrae, respectively. No significant difference $(P=.065)$ was apparent. Sensitivity and specificity of pedicle involvement for malignant pathologic fractures were $84 \%$ and $36 \%$, respectively. Among osteoporotic fractures $(n=225), 85$ vertebrae (41.3\%) showed signal-intensity changes in both T1WI and STIR images and diffuse contrast enhancement of the pedicle.

The relationship between pedicle involvement on MR imaging and the duration from the onset of pain in patients with osteoporotic compression fracture is given in Table 2. With osteoporotic fractures, pedicle involvement was seen in 84 $(77 \%)$ of 109 vertebrae with early-phase fractures $(\leq 3$ 

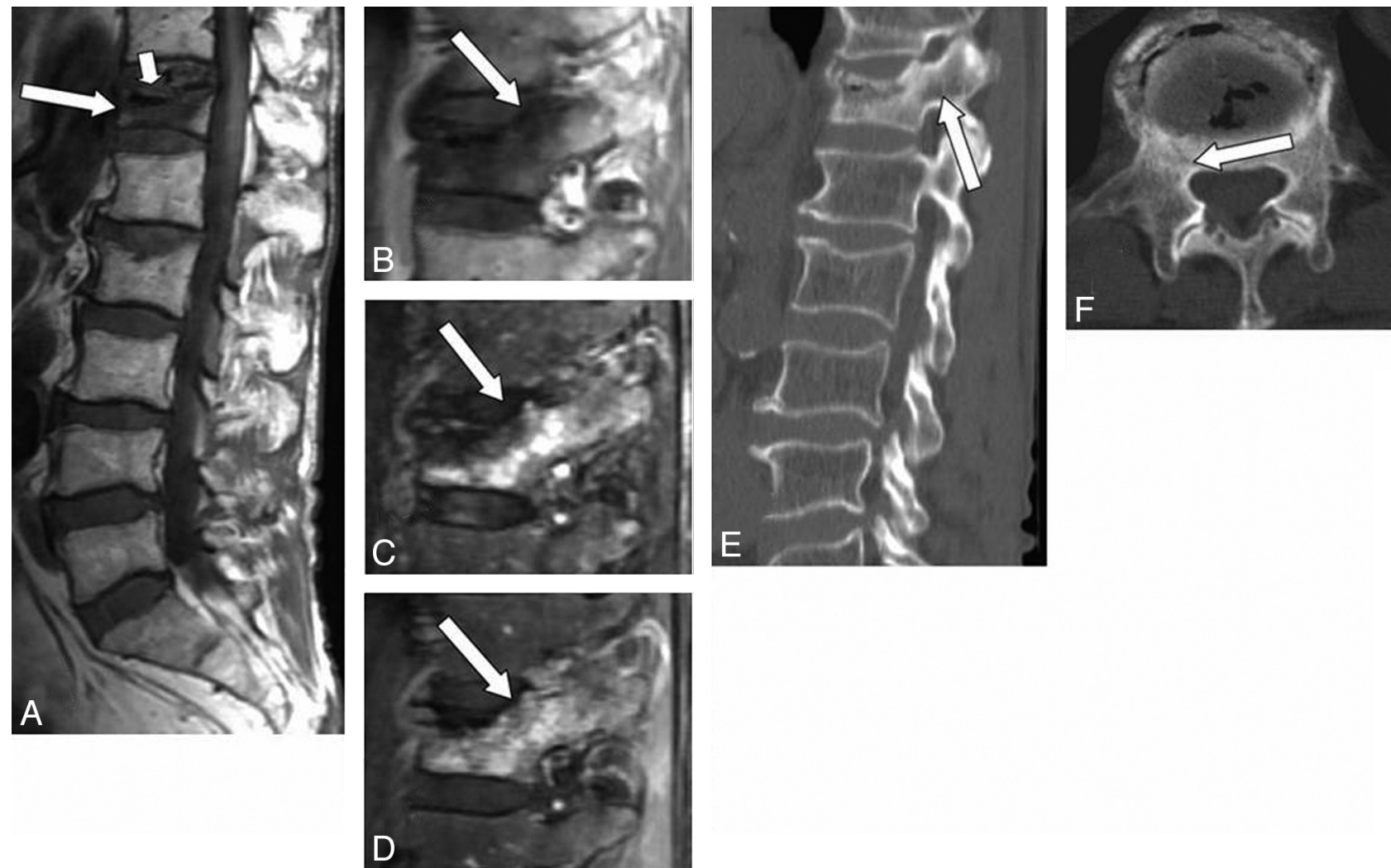

Fig 2. A 65-year-old man with benign compression fracture, which occurred 2 months earlier. $A$ and $B$, T1WI shows diffuse hypointensity in the vertebral body and the right pedicle of T12 (arrow). There is a marked hypointensity band adjacent to the superior endplate, indicating a gas cleft (small arrow). C. STIR image shows heterogeneous hyperintensity in these areas (arrow). D, Contrast-enhanced T1WI shows moderate enhancement in the vertebral body and in the right pedicle (arrow). $E$ and $F$, On sagittal $(E)$ and axial $(A C T$ scans, sclerosis is seen around the gas cleft including the base of the right pedicle (arrow).
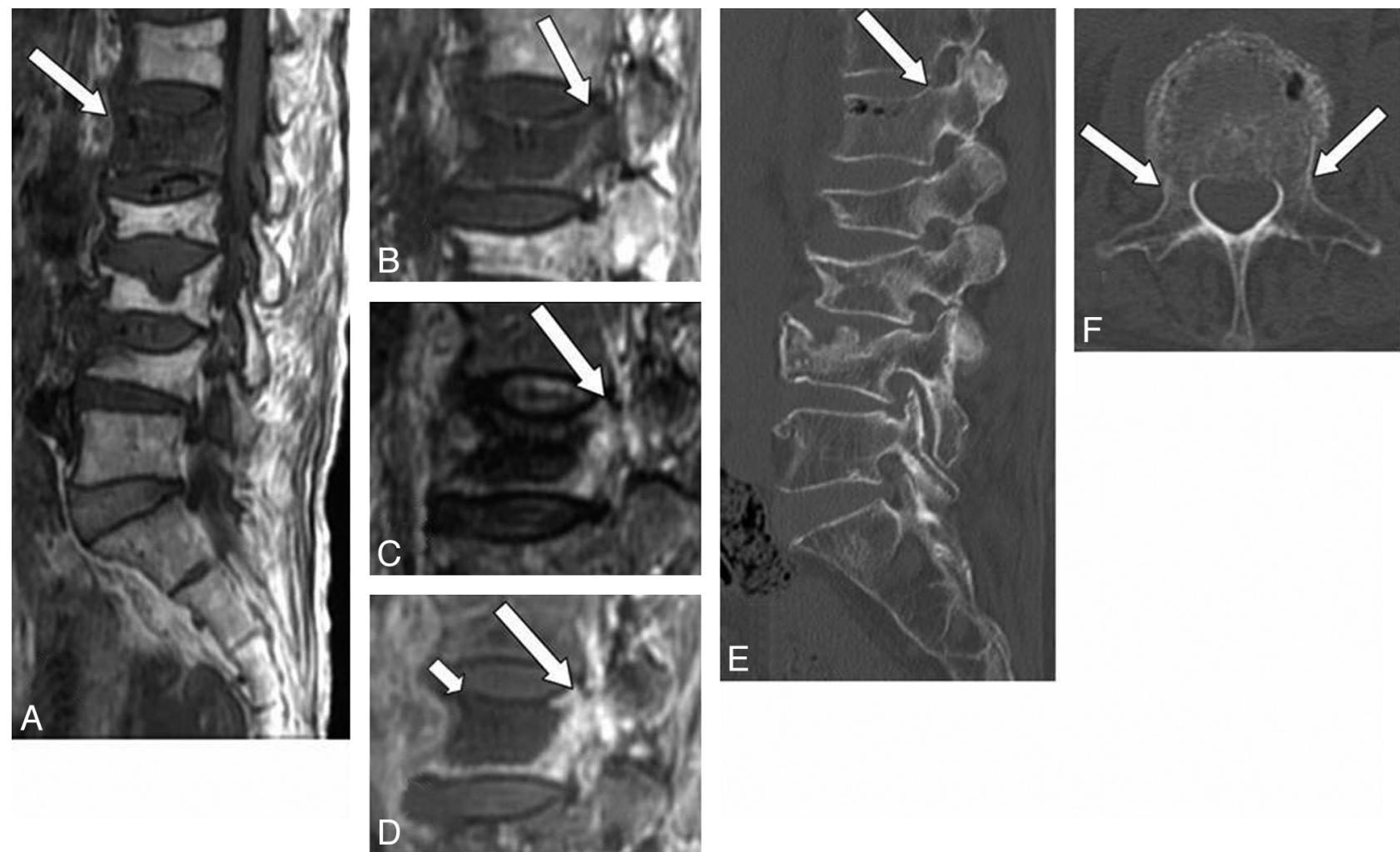

Fig 3. A 76-year-old woman with benign compression fracture, which occurred 1 month earlier. $A$ and $B$, T1WI shows diffuse hypointensity in the vertebral body and in the left pedicle of L1 (arrow). C, STIR image shows heterogeneous hyperintensity in these areas (arrow). D. Contrast-enhanced T1WI shows marked enhancement (arrow). A large area of low signal intensity indicates necrosis or cleft (small arrow). $E$ and $\mathrm{F}$, On sagittal (E) and axial (F) CT scans, there is no apparent abnormality in the pedicle (arrow). 
Table 1: Pedicle involvement in osteoporotic compression fractures (benign) and malignant pathologic fractures on MR imaging

\begin{tabular}{|c|c|c|}
\hline \multirow[b]{2}{*}{ Fracture } & \multicolumn{2}{|c|}{$\begin{array}{l}\text { Pedicle Involvement on MR } \\
\text { Imaging }\end{array}$} \\
\hline & Positive & Negative \\
\hline Benign $(n=225)$ & $144(64 \%)^{a}$ & 81 \\
\hline Malignant $(n=19)$ & $16(84 \%)^{a}$ & 3 \\
\hline
\end{tabular}

Table 2: The relationship between pedicle involvement and duration from onset of pain in patients with osteoporotic compression fractures

\begin{tabular}{lcc}
\hline & \multicolumn{2}{c}{$\begin{array}{c}\text { Pedicle Involvement on MR } \\
\text { Imaging }\end{array}$} \\
\cline { 2 - 3 } & $\begin{array}{c}\text { Positive } \\
(n=144)\end{array}$ & $\begin{array}{c}\text { Negative } \\
(n=81)\end{array}$ \\
Duration from Onset & $84(77 \%)^{\mathrm{a}}$ & 25 \\
\hline $1-3$ Months $(n=109)$ & $60(51 \%)^{\mathrm{a}}$ & 56 \\
\hline 3 Months $(n=116)$ & &
\end{tabular}

Table 3: The correlation between pedicle involvement in osteoporotic fractures and CT findings

\begin{tabular}{lcc}
\hline & \multicolumn{2}{c}{ Pedicle Involvement on MR Imaging } \\
\cline { 2 - 3 } CT Findings & $\begin{array}{c}\text { Positive } \\
(n=144)\end{array}$ & $\begin{array}{c}\text { Negative } \\
(n=81)\end{array}$ \\
\hline No findings $(n=111)$ & 44 & 67 \\
Sclerosis $(n=69)$ & 55 & 14 \\
Fracture $(n=45)$ & 45 & 0 \\
\hline
\end{tabular}

months) and 60 (51.3\%) of 116 vertebrae with chronic-phase fractures ( $>3$ months). Early-phase fractures showed a significantly larger number of patients with pedicle involvement than chronic-phase fractures did $(P<.001)$.

The correlation between pedicle involvement on MR imaging for osteoporotic fractures and CT findings is given in Table 3. In 144 osteoporotic fractures with pedicle involvement on MR imaging, sclerosis and pedicle fractures were seen in 55 vertebrae $(38.2 \%)$ and 45 vertebrae $(31 \%)$ on CT, respectively. No abnormality in the pedicle was seen in 44 vertebrae (30.5\%). MR imaging showed pedicle involvement in all patients with pedicle fracture on CT.

\section{Discussion}

Differentiating benign osteoporotic compression fractures from malignant pathologic fractures is clinically important because both occur frequently in elderly patients. For many years, various diagnostic signs on CT and MR imaging have been considered useful for such evaluation. ${ }^{1-12}$

Morphologic changes suggest malignancy when a convex posterior cortex of the vertebral body is seen due to a mass effect or epidural and/or paravertebral masses. ${ }^{1-4,8}$ Among these signs, the presence of an epidural mass has been reported as both specific and sensitive. Pedicle involvement has also been described as specific for malignant lesions. ${ }^{3}$ In addition, findings such as destruction of anterolateral or posterior cortical bone of the vertebral body or the pedicle on CT have been reported to suggest a malignant process. ${ }^{2}$

On the other hand, benign osteoporotic compression fractures show various signal-intensity patterns on MR imaging.
Early-phase fractures typically show a bandlike low signal intensity on T1WI, which is localized adjacent to the collapsed endplate. In most cases, signal-intensity changes are focal, and normal marrow is preserved in at least 1 area of the vertebral body. ${ }^{3}$ An intravertebral cleft including fluid or gas and a linear signal-intensity hyperintensity on STIR are significantly associated with osteoporotic fractures. ${ }^{6}$ These are believed to reflect avascular necrosis or a nonunionized bony gap with pseudoarthrosis. In addition, retropulsion of a posterior bone fragment is considered both sensitive and specific for osteoporotic fractures. ${ }^{2}$

The usefulness of diffusion-weighted MR imaging or chemical shift MR imaging in differentiating benign and malignant vertebral lesions has been reported recently, but the results are controversial. ${ }^{13,14}$

Correct diagnosis can be made in most cases of osteoporotic compression fracture because the useful signs mentioned above usually coexist in the collapsed vertebrae. However, in our experience, imaging features mimic malignant compression fractures in acute osteoporotic compression fractures, when the height of the vertebral body is preserved and diffuse signal-intensity changes are seen on T1WI, STIR, and GdT1WI. In such cases, a differential diagnosis is not always easy.

Because pedicle involvement is a common notion suggesting a malignant nature, we tend to diagnose malignant compression fracture when pedicle involvement is the only sign (Fig 1). We always evaluate the pedicle with CT and MR imaging before $\mathrm{PV}$, because fracture or abnormal signal-intensity changes of the pedicle may be the source of pain and instability. In such cases, pediculoplasty may be beneficial to relieve the pain. ${ }^{15-18}$ From our experience, abnormal signals of the pedicle are frequently seen on MR imaging with osteoporotic compression fractures, particularly in the early phase, and fracture of the pedicle is seen in approximately one-third of our patients.

As described in the "Results" section, abnormal signal intensity and contrast enhancement were seen in the pedicle in $64 \%$ of benign compression fractures and $84.2 \%$ of malignant pathologic fractures, with no significant difference between groups. This suggests that pedicle involvement is also common in benign osteoporotic compression fractures. These results differ from those of previous articles.

Yuh et $\mathrm{al}^{1}$ compared MR imaging findings between 84 benign fractures and 25 malignant fractures on T1WI and T2WI. They reported pedicle involvement in 22 of the 25 patients with malignant compression fractures but in none of 52 nontraumatic compression fractures, contrasting starkly with our observations. They did not perform STIR or Gd-T1WI, which could have demonstrated subtle pathologic abnormalities in the pedicle. The results might, thus, have differed if these sequences had been used. Moreover, onset of fracture and duration of pain were not described. The prevalence of pedicle involvement varies depending on the age of the fracture. Tumor involvement usually remains for a long duration if untreated, but signal intensity abnormality of the pedicle may disappear after healing of a benign compression fracture.

Cuenod et al ${ }^{3}$ compared MR imaging findings between 63 osteoporotic fractures and 30 malignant compression fractures in the acute phase ( $<2$ months). The MR imaging protocol included T1WI, T2WI, T2*WI, and Gd-T1WI without 
fat suppression. Pedicle involvement was seen in 24 malignant fractures and 4 benign fractures, and sensitivity and specificity for malignancy were $80 \%$ and $94 \%$, respectively, suggesting pedicle involvement as a specific sign for malignancy. They did not use a fat-suppression technique, which might be the reason for the low detectability of pedicle involvement in their patients with osteoporosis. Generally, detection of pedicle involvement by tumor is not difficult due to the presence of homogeneous diffuse signal-intensity changes or a mass effect, but signal-intensity changes in osteoporotic fractures are sometimes difficult to recognize because they are often focal and inhomogeneous. Bone marrow is almost completely replaced with fatty tissue in elderly patients, so fat suppression is considered necessary for the evaluation of diseased vertebrae.

Shih et $\mathrm{al}^{4}$ examined MR imaging findings for 37 patients with single-level vertebral compression fractures, including 21 patients with benign causes and 16 patients with malignancy. The imaging protocols included T1WI, T2WI with fat suppression, and Gd-T1WI with fat suppression, similar to those in the present study. They identified pedicle involvement in 6 vertebrae with benign fractures $(28.6 \%)$ and 11 vertebrae with malignant fractures $(68.8 \%)$, showing a clearly higher prevalence of pedicle involvement in benign compression fractures compared with those in the other studies mentioned above. ${ }^{1,11}$ This higher rate of pedicle involvement in osteoporotic compression fractures compared with other studies is probably due to the use of fat-suppression techniques. However, a difference from our study is seen in patient selection. They selected patients with solitary vertebral collapse as the only inclusion criterion. Symptoms of patients were not mentioned in that investigation, while we analyzed patients with acute-tochronic painful fractures. Signal-intensity changes are commonly seen in unhealed painful fractures and presumably contributed to the difference from our results.

To the best of our knowledge, no previous reports have identified correlations between the findings of pedicle involvement on MR imaging and CT. We investigated CT findings of the pedicle with or without pedicle involvement on MR imaging. Pedicle involvement in osteoporotic compression fractures exhibited 3 morphologic patterns on CT in this study: fracture, sclerotic change, and no abnormality. Fracture and sclerotic change of the pedicle were noted in $31 \%$ and $38.2 \%$ of vertebrae, respectively, in which MR imaging showed signalintensity abnormality. Laredo et $\mathrm{al}^{2}$ compared CT findings of 34 benign osteoporotic fractures with those of 32 malignant acute vertebral compression fractures. They found pedicle fracture in 3 vertebrae with osteoporotic fractures $(8.8 \%)$. That prevalence was significantly lower than that found in the present study. The reason is unclear but may be attributable to differences in the CT imager and imaging protocol because they evaluated by using axial images alone. We analyzed images with reconstructed axial, sagittal, and coronal planes with 3-mm section thickness obtained by using 16- or 64-detector row CT, which seems likely to have contributed to the higher detectability of abnormal findings in the pedicle in our series compared with that of Laredo et al. ${ }^{2}$

Sclerosis of the pedicle appears to represent reactive change or the healing process of microfracture. Signal-intensity changes on MR imaging are considered to represent the in- flammatory process, including exudation, inflammatory cell infiltration, granulation tissue, or fibrosis.

In our study, $30 \%$ of vertebrae in which abnormal signals were seen in the pedicle on MR imaging showed no obvious abnormality on CT. In those cases, the fractured vertebral body showed low signal intensity on T1WI and high signal intensity on STIR and Gd-T1WI, indicating edema and inflammatory changes. These changes presumably extended posterior to the pedicle. Our results show that abnormal signals can be seen more frequently in the pedicle with earlyphase fractures than with chronic fractures. Such signal-intensity changes are likely to disappear when the healing process is completed, as with fractures in other locations; however, a long period may be required for complete healing.

The present study had some limitations. First, diagnosis of benign or malignant fracture was mainly made on the basis of patient history and clinical presentation, and biopsy was performed in only selected patients. Some patients diagnosed with benign fracture thus could possibly have had malignant compression fractures. However, we believe such cases were unlikely and would have had little influence on the study result because no new malignancies were found in any patients at 3-month and 1-year follow-ups after PV.

Second, our study had a selection bias. We included only patients who underwent PV. It is possible that patients included had a higher likelihood of pedicle involvement than the general population because we performed PV for patients who had some signal-intensity changes on MR imaging in fractured vertebrae. So, this result may not be applicable to the larger population.

Third, due to time constraints, the MR imaging protocol for PV at our institution included only sagittal images, and we could not assess axial images in any except a few patients. Axial imaging may thus yield some additional information.

\section{Conclusions}

Pedicle involvement, which is accepted as a common indicator of malignant processes, is also frequent in patients with osteoporotic compression fractures, particularly in the early phase, and was not specific for malignancy in our study group. Although differentiating benign osteoporotic compression fractures and malignant pathologic fractures is possible in most cases, a diagnosis of malignant pathologic fracture should not be assumed when pedicle involvement is the only sign.

\section{References}

1. Yuh WT, Zachar CK, Barloon TJ, et al. Vertebral compression fractures: distinction between benign and malignant causes with MR imaging. Radiology 1989;172:215-18

2. Laredo JD, Lakhdari K, Bellaiche L, et al. Acute vertebral collapse: CT findings in benign and malignant nontraumatic cases. Radiology 1995;194:41-48

3. Cuenod CA, Laredo JD, Chevret S, et al. Acute vertebral collapse due to osteoporosis or malignancy: appearance on unenhanced and gadolinium-enhanced MR images. Radiology 1996;199:541-49

4. Shih TT, Huang KM, Li YW. Solitary vertebral collapse: distinction between benign and malignant causes using MR patterns. J Magn Reson Imaging 1999;9:635-42

5. Baur A, Stabler A, Arbogast S, et al. Acute osteoporotic and neoplastic vertebra compression fractures: fluid sign at MR imaging. Radiology 2002;225:730-35

6. Oka M, Matsusako M, Kobayashi N, et al. Intravertebral cleft sign on fatsuppressed contrast-enhanced MR: correlation with cement distribution pattern on percutaneous vertebroplasty. Acad Radiol 2005;12:992-99

7. Sugimura K, Yamasaki K, Kitagaki H, et al. Bone marrow diseases of the spine: 
differentiation with $\mathrm{T} 1$ and T2 relaxation times in MR imaging. Radiology 1987; 165:541-44

8. Baker LL, Goodman SB, Perkash I, et al. Benign versus pathologic compression fractures of vertebral bodies: assessment with conventional spin-echo, chemical-shift, and STIR MR imaging. Radiology 1990;174:495-502

9. Algra PR, Heimans JJ, Valk J, et al. Do metastases in vertebrae begin in the body or the pedicles? Imaging study in 45 patients. AJR Am J Roentgenol 1992;158:1275-79

10. An HS, Andreshak TG, Nguyen C, et al. Can we distinguish between benign versus malignant compression fractures of the spine by magnetic resonance imaging? Spine 1995;20:1776-82

11. Mehta RC, Marks MP, Hinks RS, et al. MR evaluation of vertebral metastases: T1-weighted, short-inversion-time inversion recovery, fast spin-echo, and inversion-recovery fast spin-echo sequences. AJNR Am J Neuroradiol 1995;16:281-88

12. Zajick DC Jr, Morrison WB, Schweitzer ME, et al. Benign and malignant processes: normal values and differentiation with chemical shift MR imaging in vertebral marrow. Radiology 2005;237:590-96
13. Baur A, Stabler A, Bruning R, et al. Diffusion-weighted MR imaging of bone marrow: differentiation of benign versus pathologic compression fractures. Radiology 1998;207:349-56

14. Spuentrup E, Buecker A, Adam G, et al. Diffusion-weighted MR imaging for differentiation of benign fracture edema and tumor infiltration of the vertebral body. AJR Am J Roentgenol 2001;176:351-58

15. Fuwa S, Numaguchi Y, Kobayashi N, et al. Percutaneous pediculoplasty for vertebral hemangioma involving the neural arch: a case report. Cardiovasc Intervent Radiol 2008;31:189-92

16. Eyheremendy EP, De Luca SE, Sanabria E. Percutaneous pediculoplasty in osteoporotic compression fractures. J Vasc Interv Radiol 2004;15:869-74

17. Gailloud P, Beauchamp NJ, Martin JB, et al. Percutaneous pediculoplasty: polymethylmethacrylate injection into lytic vertebral pedicle lesions. J Vasc Interv Radiol 2002;13:517-2

18. Martin JB, Wetzel SG, Seium Y, et al. Percutaneous vertebroplasty in metastatic disease: transpedicular access and treatment of lysed pedicles-initial experience. Radiology 2003;229:593-97 